J ournal of Bangladesh Chemical Society, Vol. 25(1), 71-79, 2012

\title{
MICROEMULSIONS AS NANOREACTORS FOR PREPARATION OF NANOPARTICLES WITH ANTIBACTERIAL ACTIVITY
}

SAKHAWAT HOSSAIN ${ }^{\mathrm{a}}$, UMMUL K. FATEMA ${ }^{\mathrm{a}}$, MD. YOUSUF A. MOLLAH ${ }^{\mathrm{a}}$, M. MUHIBUR RAHMAN ${ }^{b}$ AND MD. ABU BIN HASAN SUSAN ${ }^{a} *$

${ }^{a}$ D epartment of Chemistry, University of D haka, Dhaka-1000, Bangladesh

bU niversity Grants Commission of B angladesh, A gargaon, Dhaka-1207, B angladesh

\section{Abstract}

Nanoparticles of silver and copper have been synthesized by reduction of aqueous silver nitrate and copper chloride solutions with sodium borohydride in anionic water-in-oil (w/o) microemulsions, sodium dodecyl sulphate (SDS)/1-pentanol/cyclohexane/water. Scanning electron microscopic (SEM) images, energy dispersive spectra (EDX) and UVspectra have been used to characterize the resultant nanoparticles. The average size of the nanoparticles has been found to be influenced by the water to surfactant molar ratio $\left(W_{o}\right)$ in the microemulsions. Smaller particles are obtained at low $W_{o}$, while higher $W_{o}$ yields larger particles. The antibiotic sensitivity of silver and copper nanoparticles against Escherichia coli (E. coli) was tested by zone inhibition method using nanoparticles in ethanol suspension. Both silver and copper nanoparticles showed the potential for use as antibacterial agents against E. coli with sensitivity as good as conventional antibiotics. Silver nanoparticles show higher antibacterial activity against E. coli, compared to copper nanoparticles. As the amount of the nanoparticles in the suspension decreases, antibacterial activity decreases; however the concentration dependence on antibacterial activity has been less pronounced.

\section{Introduction}

Nanoparticles exhibit unique characteristics, which inter alia include unusual electronic, optical, magnetic and chemical properties that can hardly be obtained from conventional bulk materials. Their extremely small sizes and large specific surface areas associated with the ease of synthesis and chemical modification have made them most fascinating materials of current research for potential applications in diverse areas, e.g. chemistry, physics, material science, medical sciences, biology and the corresponding engineerings ${ }^{1-}$ ${ }^{3}$. In particular, there has been an upsurge of interest for application of nanoparticles of different metals and their polymer composites for biomedical applications ${ }^{4-8}$. At present, investigation of antibacterial phenomenon has regained importance due to the increase of bacterial resistance to antibiotics, caused by their overuse.

The use of silver and copper nanoparticles as antibacterial agent is relatively new. The antibacterial and antifungal properties of copper and silver are effective in reducing the growth of various microorganisms ${ }^{9-11}$. Because of their high reactivity due to the large surface to volume ratio, nanoparticles play a crucial role in inhibiting bacterial growth in aqueous and solid media. Copper nanoparticles with a larger surface to volume ratio may provide more efficient means for antibacterial activity ${ }^{12}$. The antimicrobial activity of colloidal silver particles is influenced by the dimensions of the particles, the smaller the particle size is, the greater is the antimicrobial effect ${ }^{13}$. Therefore, development of novel

\footnotetext{
*Author for Correspondence; e-mail: susan@ univdhaka.edu
} 
routes for synthesis of nanoparticales with tunable dimension has become a fascinating domain of current research.

Research to-date includes numerous attempts to prepare nanosized materials. The techniques used are gas-evaporation, sputtering, co-precipitation, sol-gel method, hydrothermal, microemulsion and so on. Microemulsions are transparent, isotropic liquid media of two mutually immiscible liquid phases (oil and water) in which one phase is dispersed in another continuous phase to bring them into a single phase (macroscopically homogeneous but microscopically heterogeneous) with the assistance of surfactants accumulated at the oil/water interface. In water-in-oil (w/o) microemulsions, nanosized water droplets (stabilized by a surfactant and dispersed in an oil phase) act as nanoreactors, wherein particle formation occurs and these droplets help to control the size of nanoparticles. Kurihara et. al. ${ }^{14}$ synthesized colloidal gold nanoparticles by reduction of chloroauric acid in water and in w/o microemulsions and found a number of advantages of forming colloidal particles in microemulsions than those obtained in homogeneous solutions. The preparation of palladium ultrafine particles by the reduction of a palladium salt with hydrazine in aerosol-T (AOT)/isooctane reverse micellar solutions has been reported by Chen and coworkers ${ }^{15}$. Ma et. al. ${ }^{16}$ employed a Triton X100 (TX-100)/hexyl alcohol/cyclohexane/water microemulsion system and prepared pure zirconia powder with the diameter lower than $10 \mathrm{~nm}$. Furthermore, TX100/pentanol/cyclohexane/ water microemulsion system was used by Tang et. al. ${ }^{17}$ for preparation of ultrafine spindle zirconia powder of monoclinic crystal structure with the diameter of 4-20 nm while employing a relatively low reactant concentration. Thus w/o microemulsions have been used as an attractive reaction medium for preparing nanoparticles, especially of metals by reduction of their salts. The highly dispersed surfactant-covered water pools offer a unique nanoenvironment for the formation of nanoparticles. They not only act as nanoreactors for processing reactions but also inhibit the excess aggregation of particles since the surfactants could adsorb on the particle surface when the particle size approaches to that of water pool. As a result, the particles obtained in such a medium are generally nanosized and monodispersed ${ }^{18}$.

Despite numerous studies on the preparation of nanoparticles using w/o microemulsions, preparation of antimicrobial nanoparticles using SDS/1-pentanol/cyclohexane/water microemulsion system has not, to our knowledge, been described in the literature. This prompted us to study the preparation of antimicrobial nanoparticles in anionic w/o microemulsions as nanoreactors and to investigate how water to surfactant molar ratio $\left(W_{o}\right)$ affects the size of the microemulsion droplets as well as size of the nanoparticles and finally to monitor the antimicrobial activity of the nanoparticles.

\section{Experimental}

\section{Materials and methods}

Sodium dodecyl sulfate (SDS), silver nitrate $\left(\mathrm{AgNO}_{3}\right)$, copper chloride $\left(\mathrm{CuCl}_{2}\right)$, sodium borohydride $\left(\mathrm{NaBH}_{4}\right)$, 1-petanol and cyclohexane were received from E. Merck and used without further purifications. Sterile nutrient agar plates, culture of bacteria (E. coli), 
cotton swab, and metric ruler (for the measurement of zone diameter) were used during antibacterial test of the prepared nanoparticles.

Synthesis of nanoparticles

Reduction of the silver and copper salts was carried out with reducing agent, $\mathrm{NaBH}_{4}$ in w/o microemulsions, comprised SDS, 1-pentanol, cyclohexane and water. The composition of the quaternary microemulsion system was varied to change the microenvironment. Table 1 lists the composition of microemulsions used for the preparation of nanoparticles. The microemulsions were prepared by mixing SDS, 1pentanol, cyclohexane followed by the addition of pure deionized water. The final mixture was a clear microemulsion system.

Table 1. Composition of microemulsion (ME) systems used for preparation of the silver and copper nanoparticles

\begin{tabular}{|c|c|c|c|c|c|}
\hline \multirow{2}{*}{ Microemulsions } & \multicolumn{4}{|c|}{$\%$ wt of } & \multirow{2}{*}{$\begin{array}{c}\text { Water to } \\
\text { surfactant molar ratio, } \\
\left(\mathrm{W}_{\mathrm{o}}\right)\end{array}$} \\
\hline & SDS & Water & Cyclohexane & 1-pentanol & \\
\hline ME-1 & 10.8 & 5.2 & 62.3 & 21.7 & 7.7 \\
\hline ME-2 & 10.2 & 10.0 & 59.3 & 20.5 & 15.7 \\
\hline ME-3 & 10.0 & 13.0 & 56.8 & 20.2 & 20.8 \\
\hline ME-4 & 9.60 & 17.2 & 54.1 & 19.1 & 28.7 \\
\hline
\end{tabular}

For the preparation of silver nanoparticles, two microemulsion systems with $\mathrm{AgNO}_{3}$ solubilized in one solution and $\mathrm{NaBH}_{4}$ as the reducing agent in the other were rapidly mixed in equal volume. The mixture instantly turned yellow in color to indicate the formation of silver nanoparticles ${ }^{19}$. In the same way copper nanoparticles were prepared by reduction of $\mathrm{CuCl}_{2}$ where the mixture instantly assumed the color of metallic copper.

Characterization of silver and copper nanoparticles

Spectral analyses were carried out in a double beam Shimadzu UV-Visible spectrophotometer, Model UV-1650 PC. Rectangular quartz cells of path length $1 \mathrm{~cm}$ were used throughout the investigation. The average particle size and morphology of the nanoparticles were investigated by scanning electron microscopy (SEM) (Model JSM6490LA). Energy dispersive X-ray (EDX) spectral analyses were also carried out (JSM6490LA) to confirm the presence of elemental silver and copper. Stubs of aluminum were used as sample stages for the SEM and EDX measurements. The accelerating voltage of $20 \mathrm{kV}$ was used for the measurements.

Antimicrobial activity measurement

One sterile nutrient agar plate was inoculated with E. coli. Ethanol suspension of colloidal silver and copper particles and their diluted solutions (10 and 100 times dilution) were dropped in different zones of the plate. The plate was then incubated at $37^{\circ} \mathrm{C}$ for 48 hours. The zone size for inhibition around each drop was determined by measuring the diameter of the zone in millimeters. 


\section{Results and Discussion}

Microemulsion systems for synthesis of nanoparticles

The synthesis of nanoparticles of silver and copper were carried out in w/o microemulsions of an anionic surfactant SDS. The aqueous cores of microemulsion systems serve as compartmentalized media (nanoscaled reactors) for chemical reactions in a controlled manner resulting in the formation of nanoparticles. The size (diameter of the core) of the nanoreactors was controlled by changing the composition of w/o of the microemulsions during the preparation of metal particles in order to control the size of the metal particles. The UV spectrum of resultant silver nanoparticles (Fig. 1) obtained in microemulsions revealed formation of nanosized particles. The spectrum shows a band around $400 \mathrm{~nm}$ which is caused by plasma resonance absorption of silver nanoparticles ${ }^{20}$. The UV spectrum of copper nanoparticles (Fig. 1) does not exhibit the expected plasmon peak due to the plasma oscillation characteristic of the copper surface. The copper particles with a diameter below $4 \mathrm{~nm}$ exhibit a strong broadening of the plasmon band ${ }^{21,}$ ${ }^{22}$. The copper particles obtained in this work have an average particle size much larger than $4 \mathrm{~nm}$ (vide infra). Accordingly, the absence of the plasmon peak could not result from broadening of the band due to the size effect. In fact, $\mathrm{Cl}^{-}$anions that can form insoluble salt with copper, $\mathrm{CuCl}$, may be strongly adsorbed on copper particles to form a monolayer of $\mathrm{CuCl}$, resulting in strong damping of the plasmon band. The results obtained from EDX spectrum ( $\mathrm{Cl}-\mathrm{K} \alpha$ at $2.621 \mathrm{KeV})$ confirmed the presence of some $\mathrm{CuCl}$ and copper particles as the main products of reduction in microemulsions. $\mathrm{Cl}^{-}$ anions can readily form insoluble $\mathrm{CuCl}$ with $\mathrm{Cu}^{+}$in w/o microemulsion media. Therefore, it is conceivable that the formation of a $\mathrm{CuCl}$ monolayer on the copper particles is responsible for the disappearance of the plasmon peak in the absorption spectrum of the copper particles obtained in this work.

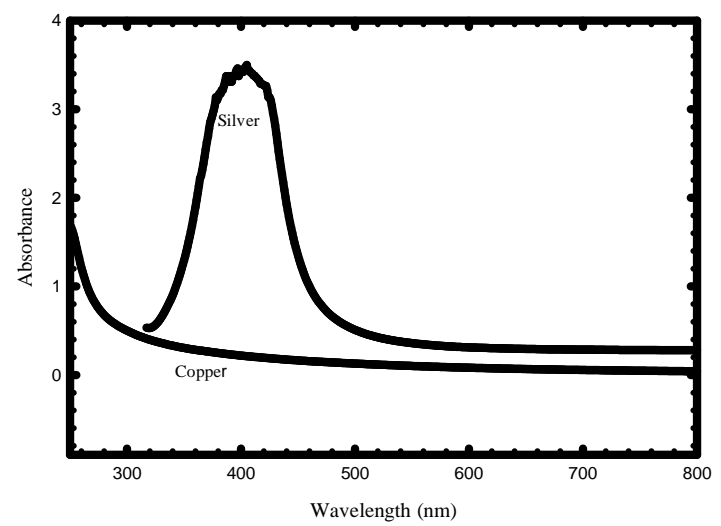

Fig. 1. Absorption spectra of the silver and copper particles obtained in SDS/1pentanol/cyclohexane/water microemulsions $\left(\mathrm{W}_{\mathrm{o}}=7.7\right)$; Precursors: For silver particles, $5 \%(\mathrm{w} / \mathrm{v})$ $\mathrm{NaBH}_{4}, 1 \%$ (w/v) $\mathrm{AgNO}_{3}$ and for copper particles, $5 \%$ (w/v) $\mathrm{NaBH}_{4}, 0.1 \mathrm{M} \mathrm{CuCl}_{2}$. 


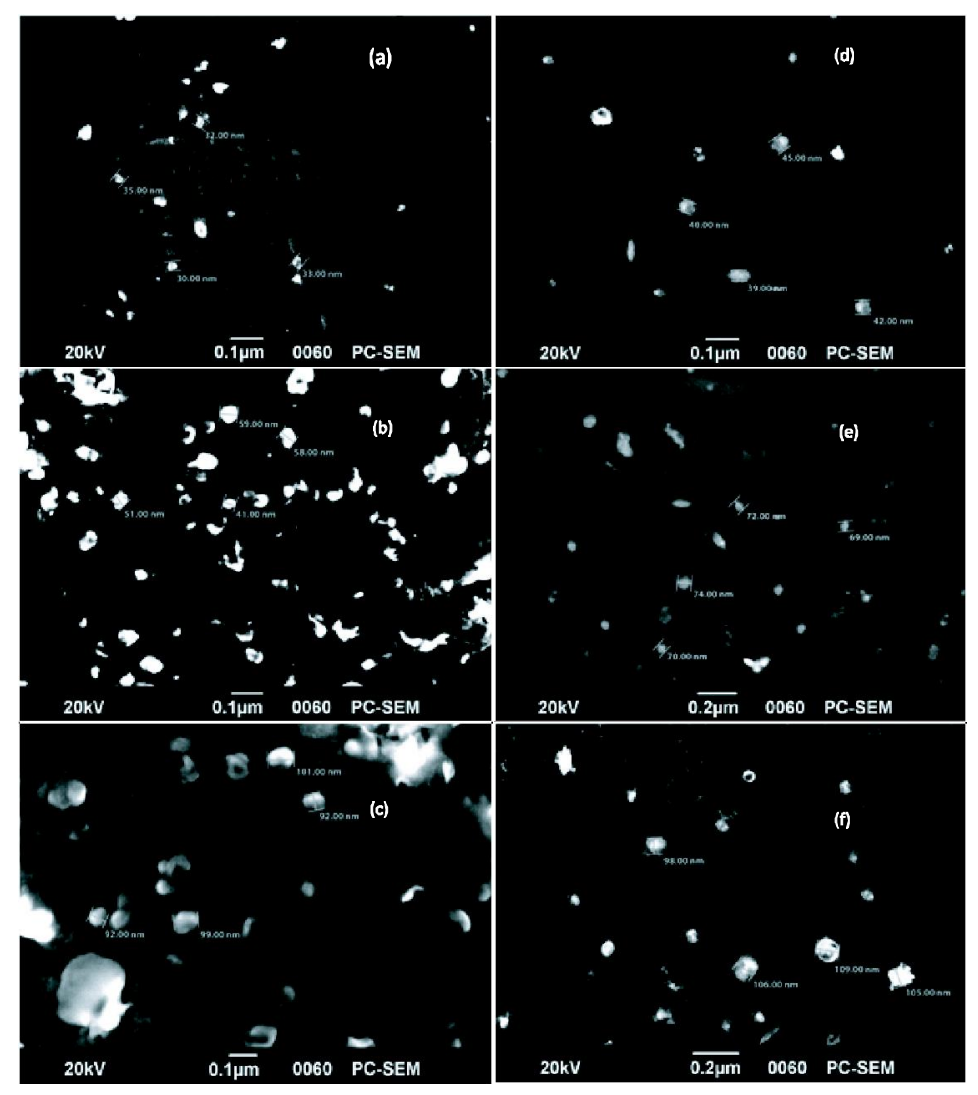

Fig. 2. SEM images of silver $(a, b, c)$ and copper $(d, e, f)$ nanoparticles obtained in SDS/1pentanol/cyclohexane/water microemulsions at different $W_{o}$ : (a), (d) 15.7; (b), (e) 20.8; (c), (f) 28.7 .

It is evident from the SEM images (Fig. 2), that silver (a,b,c) and copper (d,e,f) particles at different $W_{o}$ are formed in nanodimensions. The size of the nanoparticles can be tuned by changing composition of microemulsions. Smaller particles are obtained at low $\mathrm{W}_{\mathrm{o}}$, while the higher $W_{0}$ yields larger particles with large distribution. The shapes of silver and copper nanoparticles (Fig. 2) are spherical. The EDX spectra confirm the presence of metallic silver and copper. The spectrum shows $\mathrm{K}_{\alpha}$ peak of copper at $8.04 \mathrm{KeV}$ which is almost equal to the calculated value from $E_{\mathrm{K} \alpha}=(10.2 \mathrm{eV})(\mathrm{Z}-1)^{2}$ (where $\mathrm{Z}$ is the atomic number). Similarly, the presence of silver is observed from $\mathrm{L}$ peak of silver at $2.983 \mathrm{KeV}$.

\section{Dependence of nanoparticles size on microemulsion composition}

The size of the particles formed in the microemulsion environment bound by the micellar 'cage' is governed by the value of $W_{0}$. From the average particle size of silver and copper 
estimated from SEM images (Fig. 2), it is apparent that an increase in $W_{0}$ causes a linear increase in the particle size in the microemulsions of SDS (Fig. 3).

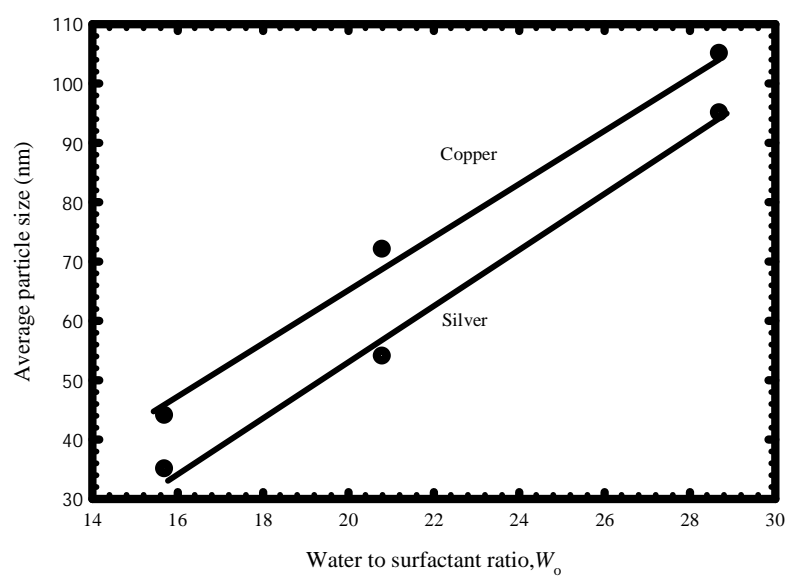

Fig. 3. Average particle size of silver and copper in SDS/1-pentanol/cyclohexane/water microemulsions as a function of $\mathrm{W}_{\mathrm{o}}$.

In fact, the amount of water in w/o microemulsions dictates the degree of interaction between the surfactant head groups and the nanoparticles, and the rigidity of the protective layer of a surfactant. Less interaction between the surfactant head groups and the nanoparticles and less surface rigidity are likely to induce aggregation of the nanoparticles. The increase in the particle size with increasing $W_{o}$ is, therefore, attributed to slow nucleation accompanied by particle growth and aggregation. Increasing the water content of the microemulsion promotes intermicellar nucleation. High water content introduces a dilution effect and reduces nucleation within individual reverse micelles. Hence, nucleation becomes dependant on the intermicellar exchange of solubilizate. In addition, high water content reduces the rigidity of the surfactant protective layer. Less rigid surface promotes particle aggregation as a result of intermicellar exchange of solubilizate and subsequently larger particles form.

Antibacterial sensitivity of silver and copper nanopartilces

The antibacterial sensitivity of silver and copper against E. coli, a gram negative bacterium commonly found in the lower intestine of warm-blooded organisms (endotherms) was tested using zone inhibition method. The diameters of inhibited zone (expressed in millimeter) by silver and copper nanoparticles at different dilutions are shown in Fig. 4. Silver shows a better antibacterial activity against E. coli (zone inhibition $20 \mathrm{~mm}$ ) than that of copper (zone inhibition $18 \mathrm{~mm}$ ). As the concentration decreased, antibacterial activity decreased indicating the concentration dependence of antibacterial activity. 


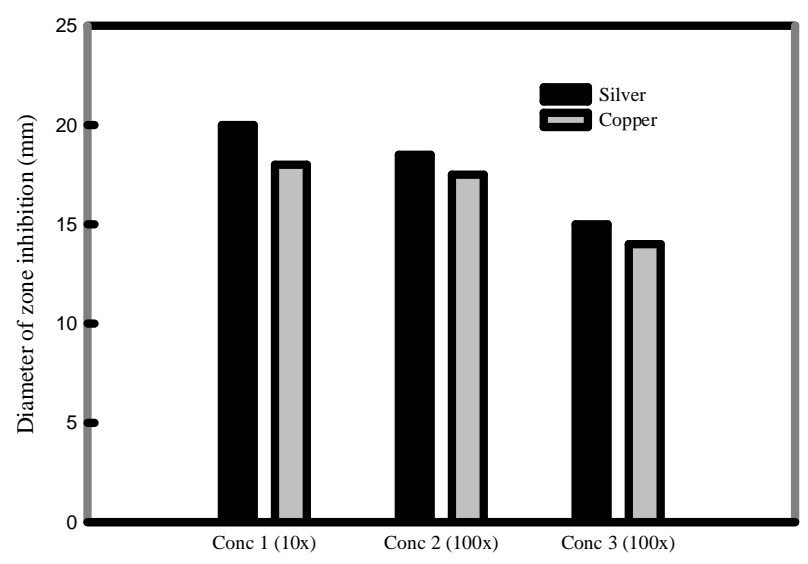

Fig. 4. Diameter of inhibited zone against E. coli for different concentration of silver and copper nanoparticles in ethanol suspensions

Fig. 5 demonstrates antibacterial sensitivity of silver and copper nanoparticles compared with conventional antibiotics. Silver nanoparticles showed better bactericidal efficiency compared to some conventional antibiotics such as ampicilin, tetracycline, gentamycin, kanamycin, streptomycin etc. for bactericidal effects on E. coli. Copper nanoparticles show almost equivalent sensitivity compared to conventional antibiotics.

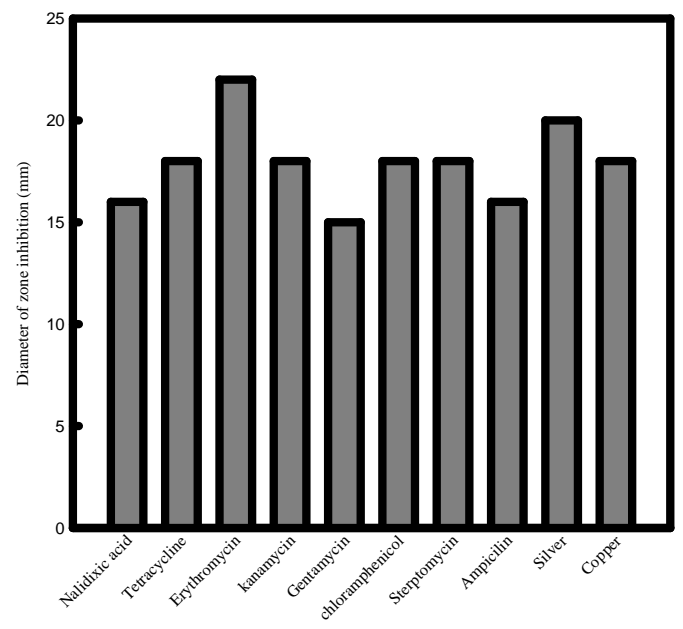

Fig. 5. A comparison of antibacterial sensitivity of silver and copper nanoparticle with conventional antibiotics. 
The mechanism by which silver and copper nanoparticles inhibit bacterial growth is not yet clearly understood and further work in this respect is in progress. However it may be explained by oligodynamic effect, a toxic effect of metals or their ions on living cells, algae, molds, spores, fungus, virus, prokaryotic and eukaryotic microorganisms, even in relatively low concentrations. Silver atoms may bind to thiol groups $(-\mathrm{SH})$ in enzymes and subsequently causing their deactivation. Silver forms stable S-Ag bonds with thiolcontaining compounds in the cell membrane that are involved in transmembrane energy generation and ion transport ${ }^{23}$. The reason for lower sensitivity of $E$. coli to the copper nanoparticles may be attributed to lower abundance of amines and carboxyl groups on cell surface of E. coli and greater affinity of copper towards these groups.

\section{Conclusion}

Silver and copper nanoparticles in anionic w/o microemulsions of water/SDS/1-pentanol/ cyclohexane have been prepared at different $W_{0}$. SEM images revealed that the average nanoparticle size was influenced by $W_{\mathrm{o}}$. Smaller particles were obtained at low $\mathrm{W}_{\mathrm{o}}$, while higher $W_{o}$ gave larger particles. The size of the naoparticles can be tuned by changing $W_{0}$ i.e. composition of microemulsions and thus suitable compositions of microemulsions may be used as nanoreactors for the preparation of nanoparticles of desired size. Both silver and copper nanoparticles have the potential to be used as alternatives to conventional antibiotics.

\section{Acknowledgement}

The authors gratefully acknowledge financial support for a sub-project (CP-231) from the Higher Education Quality Enhancement Project of the University Grants Commission of Bangladesh financed by World Bank and the Government of Bangladesh. The authors also acknowledge the facilities availed iat the Centre for Advanced Research in Sciences for SEM and EDX measurements and Molecular Genetics Lab, ICDDR,B for antibacterial activity measurements.

\section{Refer ences}

1. C. Hayashi, Phys. Today, 1987, 40, 44.

2. H. Gleiter, Prog. Mater Sci., 1989, 33, 223.

3. R. Uyeda, Prog. M ater Sci., 1991, 35, 1.

4. I. Sondi and B. Salopek-Sondi, J. Colloid Interface Sci., 2004, 275, 177.

5. F. Fermo, K. S. Morley, B.Wong, B. L. Sharp, P. L. Arnold, S. M. Howdle, R. Bayston, P. D. Brown, P.D. Winship and H. J. Reid, J. Antimicrob. Chemother ., 2004, 54, 1019.

6. S. Kim and H-J. Kim, Int. Biodeterior. Biodegrad. 2006, 57, 155.

7. P. Gong, H. Li, X. He, K. Wang, J. Hu, W. Tan, S. Zhang and X. Yang, Nanotechnology, 2007, 18, 285604. 
8. Y. P. Mamunya, H. Zois, L. Apekis, E. V. Lebedev, Powder Technol., 2004, 140, 49.

9. H. J. Lee, S. Y. Yeo and S. H. Jeong, J. Mater. Sci., 2003, 38, 2199.

10. N. Cioffi, L. Torsi , N. Ditaranto, G. Tantillo, L. Ghibelli, L. Sabbatini and Chem. Mater., 2005, 17, 5255.

11. M. Raffi, F. Hussain, T. M. Bhatti, J. I. Akhter, A. Hameed and M. M. Hasan, J. M ater. Sci. Technol., 2008, 24, 192.

12. M. Raffi, S, Mehrwan, T. M. Bhatti, J. I. Akhter, A. Hameed, W. Yawar and M. M. Hasan, Ann Microbiol., 2010, 60, 75.

13. A. R. Shahverdi, A. Fakhimi, H. R. Shahverdi and M. S. Minaian. Nonomedicine, 2007, 3,168 .

14. K. Kurihara, J. Kizling, P. Stenius and H. Janos, J. Am. Chem. Soc., 1983, 105, 2514.

15. D. H. Chen, C. C. Wang and T. C. Huang, J . Colloid Interface Sci., 1999, 210, 123; R. Birringer, Mater. Sci. Eng., 1989, 117, 33.

16. T. Ma, Y. Huang, J. Yang, J. He and L. Zhao, Int. J. M ater. Eng. Appl., 2004, 25, 515; J. B. Wright, K. Lam, D. Hansen and R. E. Burrell, Am. J . Infect. Control., 1999, $27,344$.

17. J. N. Tang, X. Z. Gong and X. C. Ma, J. Shen Zhen Univ., 2001, 18, 50; K. C. Anyaogu, A. V. Fedorov and D. C. Neckers, Langmuir, 2008, 24, 4340.

18. I. Capek, Adv. Colloid Interface Sci., 2004, 110, 49.

19. S. D. Solomon, M. Bahadory, A. V. Jeyarajasingam, S. A. Rutkowsky, C. Boritz, and L. Mulfinger, J. Chem. Educ., 2007, 84, 322.

20. K. L. Kelly, E. Coronado, L. L. Zhao and G. C. Schatz, J. Phys. Chem., 2003, B, 107, 668; K. Yoon, J. H. Byeon, J. Park and J. Hwang, Sci. Total E nviron., 2007, 373, 572.

21. I. Lisiecki and M. P. Pileni, J. Am. Chem. Soc., 1993, 115, 3887.

22. I. Lisiecki and M. P. Pileni, J. Am. Chem. Soc., 1995, 99, 5077.

23. U. Klueh, V. Wagner, S. Kelly, A. Johnson and J. D. Bryers, J. Biomed. Mater. Res-A., 2000, 53, 621 . 